\title{
Emissões de motor agrícola com o uso de diferentes tipos de diesel e concentrações de biodiesel na mistura combustível
}

\author{
Gismael Francisco Perin(1), José Fernando Schlosser ${ }^{(2)}$, Marcelo Silveira de Farias ${ }^{(2)}$, \\ Javier Solis Estrada ${ }^{(2)}$, Helen Treichel(1) e Leandro Galon ${ }^{(1)}$
}

\begin{abstract}
(1)Universidade Federal da Fronteira Sul, Campus Erechim, Rodovia ERS 135, Km 72, o 200, Caixa Postal 764, CEP $99700-970$ Erechim, RS, Brasil. E-mail: gismael@uffs.edu.br, helen.treichel@uffs.edu.br, leandro.galon@uffs.edu.br (2)Universidade Federal de Santa Maria, Avenida Roraima, o 1.000, Cidade Universitária, Camobi, CEP 97105-900 Santa Maria, RS, Brasil. E-mail: josefernandoschlosser@gmail.com, silveira_farias@hotmail.com, j.solis.estrada@gmail.com
\end{abstract}

Resumo - O objetivo deste trabalho foi quantificar o efeito do tipo de diesel e da concentração de biodiesel na mistura combustível sobre as emissões de gases por motor agrícola submetido a diferentes cargas. Foram testados dois tipos de diesel: S10, com baixo teor de enxofre; e S500, com alto teor de enxofre; bem como suas misturas com 5, 10, 15, 20, 50 e $100 \%$ de biodiesel. O motor, de quatro cilindros, turboalimentado e com 105 cv de potência máxima, foi submetido a oito modos de operação, sob diferentes rotações e torques. $\mathrm{O}$ ensaio seguiu a metodologia prevista na norma NBR ISSO 8174-4:2012, tendo-se analisado as emissões de $\mathrm{O}_{2}, \mathrm{CO}_{2}$, $\mathrm{NO}_{2}, \mathrm{NO}, \mathrm{CO}$ e de hidrocarbonetos (HC). Somente as emissões de $\mathrm{HC}$ foram influenciadas pelo tipo de diesel utilizado. As emissões de $\mathrm{NO}_{2}$ e $\mathrm{O}_{2}$ aumentaram com o aumento da concentração de biodiesel na mistura, enquanto as emissões de $\mathrm{CO}_{2}, \mathrm{NO}, \mathrm{CO}$ e $\mathrm{HC}$ diminuíram. A carga aplicada ao motor esteve diretamente relacionada às emissões, exceto para $\mathrm{O}_{2}$. O combustível $\mathrm{S} 10$, com $20 \%$ de biodiesel, reduz as emissões, em comparação ao diesel comercial (S500), sem perda no desempenho do motor.

Termos para indexação: ciclo diesel, emissão de $\mathrm{CO}_{2}$, energia renovável, freio dinamométrico, poluentes, teor de enxofre.

\section{Emissions of agricultural engine using different diesel types and biodiesel concentrations in the fuel mixture}

\begin{abstract}
The objective of this work was to quantify the effect of diesel type and of biodiesel concentrations in the fuel mixture on gas emissions from an agricultural engine subjected to different loads. Two diesel types were tested: S10, with low sulfur content; and S500, with high sulfur content; as well as their mixtures with 5, $10,15,20,50$, and $100 \%$ biodiesel. The engine, with four cylinders, turbocharged, and with 105-hp maximum power, was subjected to eight operation modes, under different rotations and torques. The assay followed the methodology set out in standard NBR ISO 8174-4:2012, and the emissions of $\mathrm{O}_{2}, \mathrm{CO}_{2}, \mathrm{NO}_{2}, \mathrm{NO}$, $\mathrm{CO}$, and hydrocarbons (HC) were analyzed. Only the $\mathrm{HC}$ emissions were affected by the type of diesel used. $\mathrm{The}^{\mathrm{NO}} \mathrm{O}_{2}$ and $\mathrm{O}_{2}$ emissions increased with greater concentration of biodiesel in the mixture, whereas the $\mathrm{CO}_{2}, \mathrm{NO}, \mathrm{CO}$, and $\mathrm{HC}$ emissions reduced. The load applied to the engine was directly related to the emissions, except for $\mathrm{O}_{2}$. The S10 fuel, with 20\% biodiesel, reduces emissions, when compared to the commercial diesel (S500), without losing engine performance.
\end{abstract}

Index terms: diesel cycle, $\mathrm{CO}_{2}$ emission, renewable energy, dynamometer brake, pollutants, sulfur content.

\section{Introdução}

O uso de tecnologias de ponta nos processos mecanizados é importante para eficiência econômica e produtiva da agricultura. Atualmente, a maioria das máquinas utilizadas no meio rural é dotada de motores de ciclo diesel, por causa de sua eficiência e confiabilidade. Assim, a melhoria na eficiência desses motores ou até a adoção de novos combustíveis é necessária para reduzir os impactos ambientais e os custos das atividades agrícolas.

Nesse contexto, os biocombustíveis têm atraído grande atenção internacional e nacional, principalmente em razão de instabilidades no preço do petróleo e da discussão mundial sobre a diminuição das emissões de $\mathrm{CO}_{2}$ pelo uso de energias renováveis (Kohlhepp, 2010; Chauhan et al., 2013). Ao longo dos últimos 20 anos, houve aumento substancial nas pesquisas na área de 
biocombustíveis. Aspectos ambientais, econômicos, políticos, culturais e técnicos relativos ao uso dos biocombustíveis têm sido comumente abordados, especialmente na agricultura (Buyukkaya, 2010; Bernardes, 2011; Ballesteros et al., 2014).

No Brasil, são disponibilizados dois tipos de combustível diesel: o S10 (10 ppm), com baixo teor de enxofre; e o S500 (500 ppm), com alto teor de enxofre; esses combustíveis são utilizados nas máquinas agrícolas em misturas com 5\% de biodiesel (B5). Diante do grande interesse no uso de combustíveis menos poluentes, sem que haja redução significativa no desempenho dos motores, vários trabalhos têm sido desenvolvidos com motores diesel para uso veicular, com o objetivo de verificar o desempenho desses motores e as suas emissões de gases quando movidos por diferentes combustíveis (Dwivedi et al., 2011; Brijesh \& Sreedhara, 2013; Ballesteros et al., 2014). É comum que o biodiesel misturado no diesel mineral reduza a emissão de hidrocarbonetos e óxido de carbono, bem como a densidade de fumaça (Koçak et al., 2007; Buyukkaya, 2010), e aumente as emissões de óxido de nitrogênio, em comparação ao diesel mineral (Canakci, 2007; Sahoo et al., 2009). Além disso, o uso de maiores proporções de biodiesel no diesel mineral também tem implicações econômicas, uma vez que o biodiesel é mais caro e sua disponibilidade ainda é pequena. Apesar da abundância de trabalhos para avaliar as emissões por motores veiculares, ainda são escassos os com motores de ciclo diesel agrícolas.

O objetivo deste trabalho foi quantificar a influência do tipo de diesel e da concentração de biodiesel na mistura combustível sobre as emissões de gases por motor agrícola submetido a diferentes cargas.

\section{Material e Métodos}

O ensaio foi realizado com trator Massey Ferguson, modelo MF 4291 (AGCO, Duluth, GA, EUA), fabricado em 2012, com 73 horas de uso, equipado com motor Perkins, modelo 1104A-44T (Perkins Engines Company Limited, Peterborough, Reino Unido), de ciclo diesel de quatro cilindros, cilindrada total de $4.400 \mathrm{~cm}^{3}$ e turboalimentado. Segundo o fabricante, o motor subministra 77,2 kW (105 cv) de potência máxima, a $2.200 \mathrm{rpm}$, e $400 \mathrm{Nm}$ de torque máximo, a $1.400 \mathrm{rpm}$.

Para a aplicação de cargas parciais ao motor, foi utilizado freio dinamométrico móvel Eggers, modelo PT 301 MES (KL-Maschinenbau GmbH \& Co. kg,
Rendsburg, Alemanha), de correntes de Foucault, refrigerado a ar, com capacidade de frenagem de até $600 \mathrm{~kW}$, com acoplamento pela tomada de potência (TDP) do trator. O controle das cargas aplicadas e o cálculo da potência utilizada foram feitos com uso de programa específico e da coleta dos dados de torque e rotação do motor. Para a quantificação das emissões, utilizou-se o analisador de gases Saxon, modelo Infralyt ELD (Saxon Junkalor GmbH, Dessau-Roßlau, Alemanha), com capacidade de medir alguns dos principais gases de escape provenientes do motor. Esse equipamento mede $\mathrm{O}_{2}$ e $\mathrm{CO}_{2}$, em percentagem volumétrica, e $\mathrm{NO}_{2}, \mathrm{CO}, \mathrm{NO}$ e hidrocarbonetos $(\mathrm{HC})$, em ppm, nos gases de escape.

O biodiesel utilizado foi obtido pela rota metílica, tendo-se utilizado $60 \%$ de soja, $35 \%$ de gordura bovina e 5\% de gordura suína. O diesel S10 e o S500 foram adquiridos na rede de abastecimento automotiva local.

Os 11 combustíveis avaliados foram obtidos com o uso dos dois tipos de diesel (S10 e S500) e de suas misturas com diferentes proporções de biodiesel: S10B5, diesel S10 com 5\% de biodiesel; S10B10, diesel S10 com 10\% de biodiesel; S10B15, diesel S10 com $15 \%$ de biodiesel; S10B20, diesel S10 com $20 \%$ de biodiesel; S10B50, diesel S10 com 50\% de biodiesel; S500B5, diesel S500 com 5\% de biodiesel; S500B10, diesel S500 com 10\% de biodiesel; S500B15, diesel S500 com 15\% de biodiesel; S500B20, diesel S500 com 20\% de biodiesel; S500B50, diesel S500 com $50 \%$ de biodiesel; e B100, biodiesel puro (100\%).

Realizou-se, inicialmente, uma avaliação do desempenho do motor em cada tratamento. Os valores de torque nominal e máximo, bem como a rotação nominal, a de torque máximo e a de marcha lenta, são imprescindíveis na determinação das emissões, de acordo com a norma NBR ISO 8178:2012 (Associação Brasileira de Normas Técnicas, 2012). Esta norma determina que as emissões devem ser coletadas em oito pontos, isto é, em oito modos de operação, com diferentes rotações e torques. As coletas são realizadas sob três rotações: rotação nominal, com quatro pontos de coleta (modos 1 a 4); rotação de torque máximo, com três pontos de coleta (modos 5 a 7); e rotação de marcha lenta, com um ponto de coleta (modo 8). No modo 1 (M1), aplica-se uma carga equivalente a $100 \%$ do torque na rotação nominal; no $\mathrm{M} 2$, uma equivalente a $75 \%$; no M3, a $50 \%$; e no M4, a $10 \%$. Já no M5, aplica-se $100 \%$ do torque obtido na rotação de torque 
máximo; no M6, 75\%; e no M7, 50\%. Finalmente, no M8, o motor é avaliado com rotação de marcha lenta e não se aplica carga, ou seja, não há torque (Associação Brasileira de Normas Técnicas, 2012).

$\mathrm{O}$ aquecimento do motor foi monitorado por um período de $20 \mathrm{~min}$, até que se estabilizassem seus parâmetros, sob condição de carga equivalente à redução de $20 \%$ na rotação de máxima aceleração. Os ensaios foram realizados em sequência crescente dos modos de operação, do M1 ao M8, para cada combustível utilizado. O tempo de coleta dos gases de escape, em cada modo, foi de 2 min, com descarte dos dados do primeiro terço da coleta, para estabilização dos equipamentos de medição. Os dados foram coletados a cada $2 \mathrm{~s}$, com aproximadamente 40 observações para cada modo de operação e para cada combustível, o que totalizou mais de 3.500 observações.

$\mathrm{O}$ nível de emissão de $\mathrm{O}_{2}$ em função do combustível foi ajustado por meio de regressão linear com as percentagens de biodiesel na mistura, independentemente do modo de operação. Em seguida, o ajuste foi feito com os diferentes modos de operação, independentemente do combustível utilizado.

A temperatura atmosférica durante a realização do experimento aumentou de 24,5 para $30,5^{\circ} \mathrm{C}$. $\mathrm{Na}$ proporção inversa $(\mathrm{r}=-0,99 \mathrm{com}$ a temperatura), a umidade relativa do ar diminuiu de 69 para $46 \%$. Como a pressão atmosférica permaneceu praticamente inalterada com o aumento da temperatura (passou de 100,33 para $100,05 \mathrm{kPa}$; $\mathrm{r}=-0,81$ ), constatou-se que a quantidade de água na atmosfera manteve-se constante ao longo do ensaio.

As análises estatísticas foram realizadas com o programa R (R Development Core Team, 2012), tendose utilizado o pacote "Agricolae" (Mendiburu, 2012) para comparação das médias entre os tratamentos. A análise estatística consistiu da análise de variância, $\mathrm{e}$, quando o teste $\mathrm{F}$ indicou diferença significativa entre os tratamentos, as médias foram comparadas pelo teste de Tukey, a 5\% de probabilidade. Além disso, realizou-se análise de regressão para descrever o comportamento das emissões em função da variação no teor de biodiesel na mistura combustível.

\section{Resultados e Discussão}

Tanto os combustíveis como os modos de operação tiveram efeito significativo $(\mathrm{p}<0,05)$ sobre as emissões de gases. Quanto ao $\mathrm{O}_{2}$, todos os combustíveis avaliados diferiram entre si: o S500B50 foi o que apresentou a maior emissão do gás, e o S500B15, a menor (média de todas as observações), independentemente do modo de operação (Figuras 1 e 2). Todos os modos de operação também diferiram significativamente entre si: o M5 foi o que apresentou a menor emissão de $\mathrm{O}_{2}(8 \%)$, e o M8, a maior (18\%), independentemente do tipo de combustível utilizado (Tabela 1).

Os níveis de $\mathrm{O}_{2}$ emitido permaneceram constantes, e a variação com a adição de $10 \%$ de biodiesel na mistura foi de: $0,01 \%$ (coeficiente angular da equação de regressão: $\beta=0,001$ ) para o diesel S10 (Figura 1); e $0,03 \%(\beta=0,003)$ para o diesel S500 (Figura 2). A maior emissão de $\mathrm{O}_{2}$ com o diesel S500 deve-se ao seu maior conteúdo de impurezas; já seu coeficiente angular mais elevado é atribuído à adição do biodiesel às misturas, que, por ser uma substância mais pura, altera, com maior intensidade, o diesel mais impuro. Além disso, a quantidade de oxigênio presente no biodiesel é de, aproximadamente, 10 a $12 \%$, dependendo do tipo de óleo vegetal extraído, e, no diesel, de origem mineral, sua concentração é nula (Boldaji et al., 2011).

$\mathrm{O}$ comportamento da emissão de $\mathrm{O}_{2}$ em função do modo de operação está descrito na Tabela 1. Neste caso, o tipo de combustível foi desconsiderado, e utilizou-se a média das emissões para cada modo de operação. $\mathrm{O}$ ordenamento dos modos de operação foi o seguinte: M5, M1, M6, M2, M7, M3, M4 e M8. O critério utilizado para este ordenamento foi a intensidade das cargas aplicadas $(100,75,50,10 \%$ e sem carga) e a quantidade de ar admitido (da menor para a maior). Esse ordenamento está de acordo com o incremento ou a redução nos níveis de emissão das sete variáveis resposta analisadas. Para a regressão das emissões de $\mathrm{O}_{2}$, de acordo com esse ordenamento na carga do motor, o coeficiente de determinação $\left(\mathrm{R}^{2}\right)$ foi de $95 \%$.

Quanto maior a carga aplicada ao motor e menor a admissão de ar pela sua menor rotação, menor foi a emissão de $\mathrm{O}_{2}$. Isso porque a maior parte do $\mathrm{O}_{2}$ admitido ( $\sim 21 \%$ do ar) é utilizada para a queima do combustível. No modo de operação M5, $\sim 40 \%$ do $\mathrm{O}_{2}$ sobra no processo de combustão, enquanto, no $\mathrm{M} 8$, apenas $15 \%$ do $\mathrm{O}_{2}$ é utilizado nesse processo. Portanto, o modo de operação foi responsável por variações de até $45 \%$ nas emissões de $\mathrm{O}_{2}$, e o tipo 
de combustível foi responsável por apenas $2 \%$ de variação (Figuras 1 e 2).

Os HC, produzidos durante o processo de combustão ou já presentes no combustível, são compostos basicamente por hidrogênio e carbono. Entretanto, suas emissões ficam na casa das dezenas de partes por milhão (ppm) apenas. As emissões de $\mathrm{HC}$ foram mais sensíveis ao tipo de combustível do que ao modo de operação. Os combustíveis S10B5, S10B10 e S500B20 não diferiram estatisticamente entre si e foram os que mais emitiram $\mathrm{HC}$. Na sequência, os combustíveis S10B15, S10B20, S500B5 e S500B50, nesta ordem, emitiram mais HC, e o combustível B100 foi o que menos os emitiu. Na Figura 1, percebe-se que o comportamento das emissões com o diesel S10 foi linear, de acordo com a alteração na concentração de biodiesel. Houve decréscimo de 7,9 ppm nas emissões de $\mathrm{HC}$ com a inclusão de $10 \%$ de biodiesel $(\beta=0,79)$. Já com o diesel S500, o comportamento das emissões de $\mathrm{HC}$ foi quadrático (Figura 2).

Independentemente do diesel utilizado, as emissões de $\mathrm{HC}$ variaram de $72 \mathrm{ppm}$, na menor concentração de biodiesel (B5), a 8 ppm, em média, com biodiesel puro. Isso representa redução expressiva, de $88,9 \%$, nas
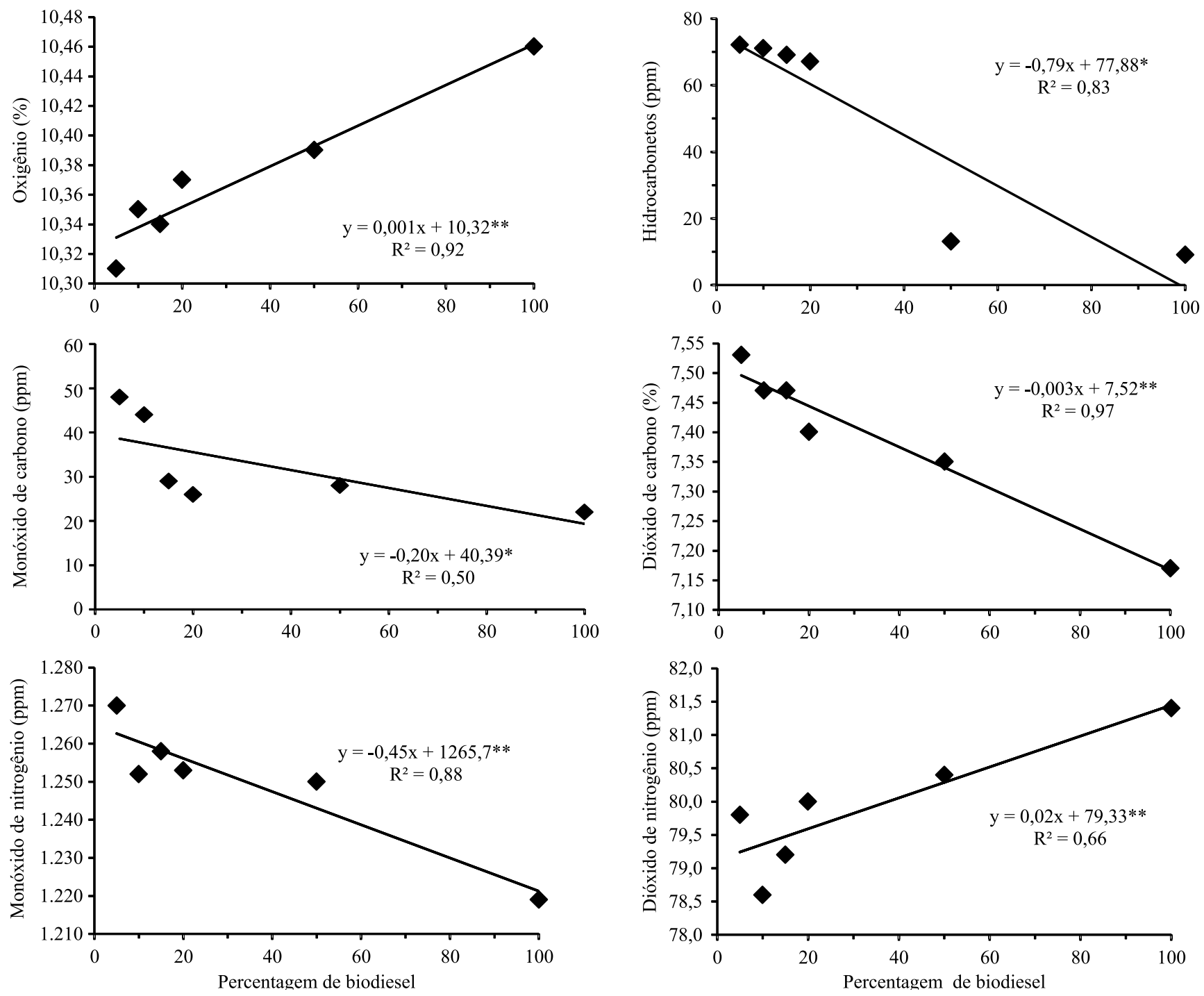

Figura 1. Emissões dos gases de escape em função do incremento do teor de biodiesel na mistura com o diesel S10, independentemente do modo de operação do motor. 
emissões. Gonzalez Maziero et al. (2006) observaram redução de $31 \%$ nos níveis de emissão de $\mathrm{HC}$, quando trabalharam com biodiesel de girassol. Já Canakci \& Van Gerpen (2003) verificaram redução de $42 \%$ com biodiesel de soja e de $46 \%$ com gordura animal. Estes autores concluíram que a diferença nas emissões de $\mathrm{HC}$ em função da fonte de biodiesel é menor do que a observada entre os níveis de mistura dos tipos de biodiesel com o diesel. Ozsezen et al. (2009) relataram redução de $21 \%$ nas emissões de HC com o incremento da concentração de biodiesel nas misturas. Por sua vez, Sanjid et al. (2014) constataram redução nas emissões de $3,7 \%$, com a inclusão de $5 \%$ de biodiesel, e de
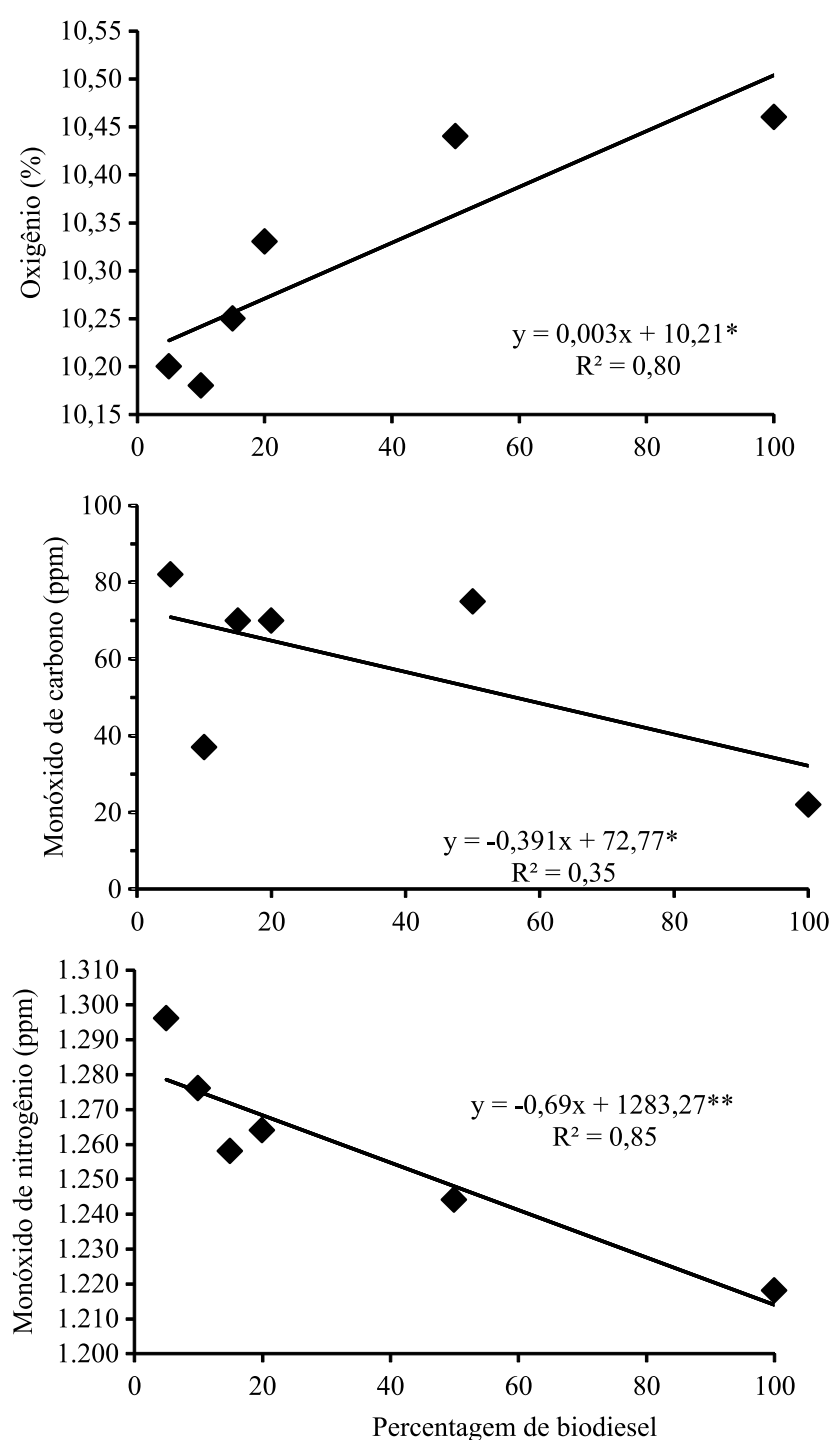

$7,8 \%$ com a inclusão de $10 \%$. McCarthy et al. (2011) reportaram que a fonte do biodiesel foi responsável por variações entre 10 e $80 \%$, nas emissões de $\mathrm{HC}$.

Houve redução nas emissões de $\mathrm{HC}$ à medida que a carga no motor diminuiu, uma vez que a admissão de ar aumentou pela maior rotação do motor (Tabela 1). Esse mesmo comportamento ocorreu, com maior ou menor intensidade, com todas as variáveis analisadas. Nesse sentido, Valente et al. (2012) também verificaram que as emissões de $\mathrm{HC}$ aumentaram com o aumento na carga aplicada ao motor. No presente trabalho, as emissões reduziram de 54,1 ppm, no M5, para 35,6 ppm, no M8, o que, em termos percentuais, representou $34,5 \%$.
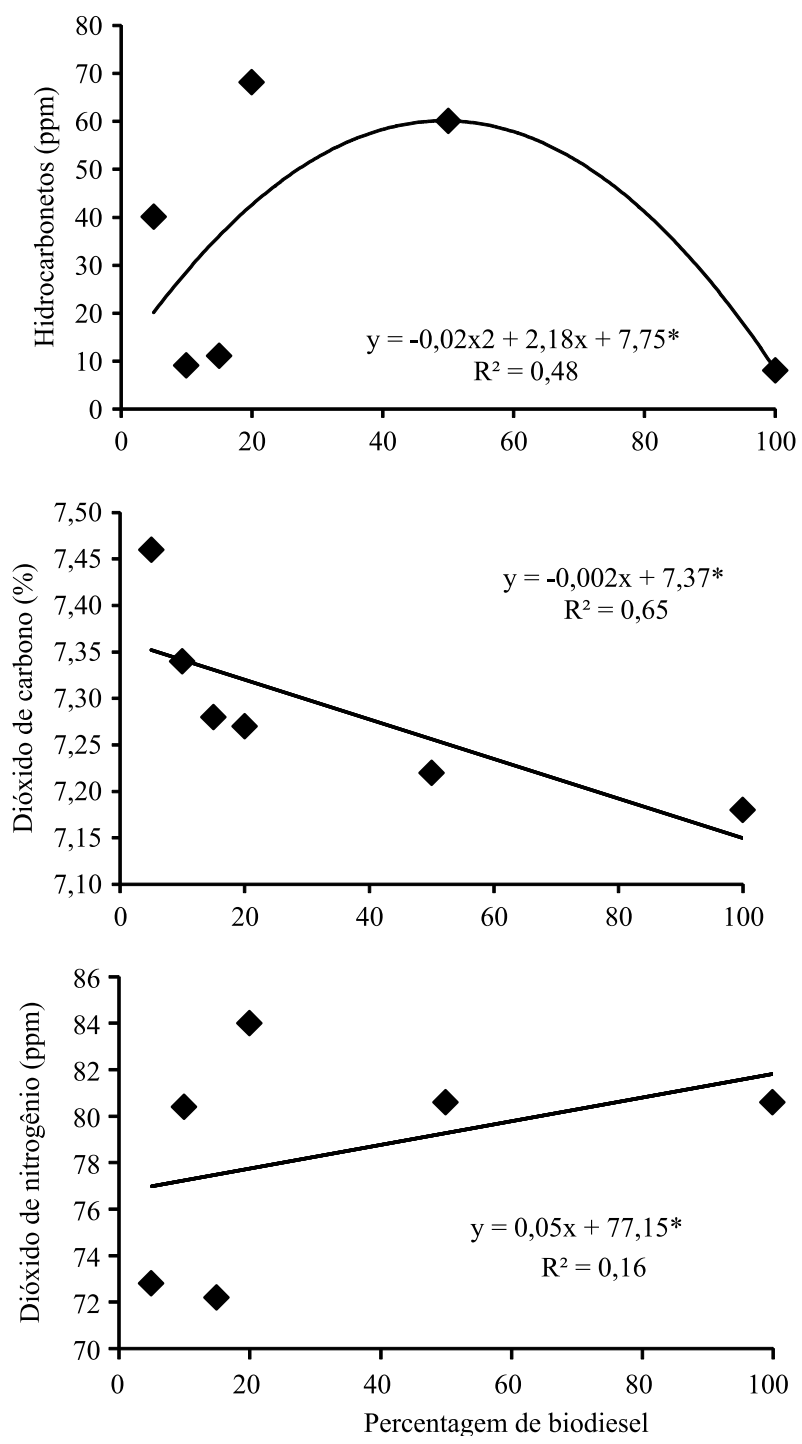

Figura 2. Emissões dos gases de escape em função do incremento do teor de biodiesel na mistura com o diesel S500, independentemente do modo de operação do motor. 
Dessa forma, no caso de HC, o tipo de combustível apresentou maior influência nas emissões do que o modo de operação, diferentemente do observado para as demais variáveis avaliadas.

As emissões de $\mathrm{CO}$ seguiram a mesma ordem de grandeza das de $\mathrm{HC}$ (ppm). O combustível que mais emitiu esse gás foi o S500B5, seguido do S500B50. Os combustíveis S500B20 e S500B15 foram os terceiros que mais emitiram $\mathrm{CO}$, não tendo diferido estatisticamente entre si. Já os combustíveis que utilizaram o diesel S10 emitiram, em média, menos CO. O biodiesel puro foi o que apresentou menor emissão (média de $22 \mathrm{ppm}$ ). As emissões de $\mathrm{CO}$ também diminuíram com o incremento da inclusão de biodiesel na mistura (Figuras 1 e 2). Esta diminuição foi mais acentuada com o diesel S500, tendo-se observado redução de 2 ppm com a adição de $10 \%$ de biodiesel no diesel S10 (Figura 1), e de 4 ppm, no diesel S500 (Figura 2).

Independentemente do tipo de diesel utilizado, houve expressiva redução $(73,2 \%)$ nas emissões de $\mathrm{CO}$ quando se utilizou o biodiesel puro, em comparação ao diesel comercial atual (B5). Se o diesel com menor teor de enxofre for considerado, essa redução foi de $54,2 \%$. McCarthy et al. (2011) relataram redução nas emissões de CO na ordem de 43\%, com o uso de biodiesel. Já Gonzalez Maziero et al. (2006) constataram que o biodiesel de girassol diminuiu em $32,2 \%$ os níveis

Tabela 1. Emissões dos gases de escape do motor de acordo com os diferentes modos de operação, independentemente do combustível utilizado ${ }^{(1)}$.

\begin{tabular}{lcccccc}
\hline $\begin{array}{l}\text { Modo de } \\
\text { operação }\end{array}$ & $\begin{array}{c}\mathrm{O}_{2} \\
(\%)\end{array}$ & $\begin{array}{c}\mathrm{HC}(3) \\
(\mathrm{ppm})\end{array}$ & $\begin{array}{c}\mathrm{CO} \\
(\mathrm{ppm})\end{array}$ & $\begin{array}{c}\mathrm{CO}_{2} \\
(\%)\end{array}$ & $\begin{array}{c}\mathrm{NO} \\
(\mathrm{ppm})\end{array}$ & $\begin{array}{c}\mathrm{NO}_{2} \\
(\mathrm{ppm})\end{array}$ \\
\hline M5 & $8,9 \mathrm{~h}$ & $54,1 \mathrm{a}$ & $762,7 \mathrm{a}$ & $8,3 \mathrm{a}$ & $1.416,4 \mathrm{a}$ & $102,6 \mathrm{a}$ \\
M1 & $10,4 \mathrm{~g}$ & $45,1 \mathrm{c}$ & $53,2 \mathrm{~cd}$ & $7,3 \mathrm{~b}$ & $1.206,8 \mathrm{c}$ & $74,6 \mathrm{c}$ \\
M6 & $11,0 \mathrm{f}$ & $46,2 \mathrm{~b}$ & $106,6 \mathrm{~b}$ & $6,9 \mathrm{c}$ & $1.280,9 \mathrm{~b}$ & $97,6 \mathrm{~b}$ \\
M2 & $12,2 \mathrm{e}$ & $42,7 \mathrm{~d}$ & $34,5 \mathrm{e}$ & $6,2 \mathrm{~d}$ & $854,3 \mathrm{~d}$ & $54,9 \mathrm{e}$ \\
M7 & $13,0 \mathrm{~d}$ & $42,9 \mathrm{~d}$ & $41,0 \mathrm{de}$ & $5,6 \mathrm{e}$ & $786,9 \mathrm{e}$ & $60,8 \mathrm{~d}$ \\
M3 & $13,8 \mathrm{c}$ & $40,8 \mathrm{e}$ & $46,3 \mathrm{de}$ & $5,0 \mathrm{f}$ & $482,0 \mathrm{f}$ & $37,7 \mathrm{f}$ \\
M4 & $16,4 \mathrm{~b}$ & $38,1 \mathrm{f}$ & $64,5 \mathrm{c}$ & $3,2 \mathrm{~g}$ & $278,0 \mathrm{~g}$ & $27,6 \mathrm{~g}$ \\
M8 & $18,5 \mathrm{a}$ & $35,6 \mathrm{~g}$ & $13,2 \mathrm{f}$ & $1,5 \mathrm{~h}$ & $161,8 \mathrm{~h}$ & $24,1 \mathrm{~h}$ \\
\hline $\mathrm{CV}(\%)$ & 2,0 & 4,8 & 56,6 & 2,8 & 4,8 & 7,1 \\
\hline
\end{tabular}

${ }^{(1)}$ Médias seguidas de letras iguais não diferem pelo teste de Tukey, a 5\% de probabilidade. ${ }^{(2)} \mathrm{M} 1, \mathrm{M} 2$, M3 e M4, carga aplicada equivalente a 100, 75, 50 e $10 \%$, respectivamente, do torque na rotação nominal do motor; M5, M6 e M7, 100, 75 e 50\%, respectivamente, do torque obtido na rotação de torque máximo; e M8, rotação de marcha lenta, sem aplicação de carga, isto é, não há torque. ${ }^{(3)} \mathrm{HC}$, hidrocarbonetos. de emissão de CO, enquanto Gokalp et al. (2011) observaram redução de 3 a $52 \%$ nas emissões do gás, com a inclusão de 5 ou $100 \%$ de biodiesel nas misturas. Özener et al. (2014) verificaram redução de até $46 \%$ nas emissões de CO com o uso da mistura B50, enquanto Sanjid et al. (2014) relataram reduções de $9,5 \%$, com B5, e 20,5\% com B10. Valente et al. (2012), no entanto, observaram aumento de $20,1 \%$ nas emissões de CO, ao usar mistura com 50\% de biodiesel de óleo de fritura.

O modo de operação M5 apresentou elevada emissão de CO (762,7 ppm), 715\% maior que a do M6, que foi o modo de operação com a segunda maior emissão do gás. Os demais modos de operação permaneceram com valores de emissão abaixo de 100 ppm, e seguiram a tendência de diminuição das emissões de CO de acordo com a redução na carga aplicada ao motor. Houve redução de $98,2 \%$ nas emissões de $\mathrm{CO}$ entre o modo de operação que mais emitiu o gás (M5) e o que menos o emitiu (M8). Valente et al. (2012) afirmam que, de fato, as emissões de $\mathrm{CO}$ diminuem com a redução na carga aplicada ao motor. Apesar do modo de operação ter resultado em maior alteração nos níveis de emissão de $\mathrm{CO}$, o tipo de combustível também apresentou influência expressiva sobre as emissões desse poluente. A influência positiva da inclusão de biodiesel no combustível sobre as emissões do CO somente foi menor do que a observada para os $\mathrm{HC}$.

As emissões de $\mathrm{CO}_{2}$ apresentaram comportamento oposto ao das emissões de $\mathrm{O}_{2}(\mathrm{r}=-0,97)$, o que era esperado. Como o $\mathrm{CO}_{2}$ produzido pela combustão é decorrente quase que exclusivamente da união do $\mathrm{O}_{2}$ atmosférico ao carbono presente no combustível (o percentual de $\mathrm{CO}_{2}$ atmosférico é pequeno, $\sim 0,04 \%$ ), os níveis de emissão de $\mathrm{CO}_{2}$ não podem ser maiores que $21 \%$ (percentual de $\mathrm{O}_{2}$ atmosférico). Além da ligação com o carbono, para a formação do $\mathrm{CO}_{2}$ e do $\mathrm{CO}$, o $\mathrm{O}_{2}$ também se liga ao $\mathrm{N}$ e forma óxido de nitrogênio $\left(\mathrm{NO}_{\mathrm{x}}\right)$, porém em menores quantidades, na escala de ppm. A quantidade de $\mathrm{CO}_{2}$ emitida entre os diferentes combustíveis e modos de operação variou de $\sim 1$ a $9 \%$.

$\mathrm{O}$ combustível que mais emitiu $\mathrm{CO}_{2}$ foi o $\mathrm{S} 10 \mathrm{~B} 10$, seguido do S10B5 e do S10B15, que não diferiram entre si. Os que menos emitiram o gás foram o S500B50, o B100 e o S10B50.

A emissão de $\mathrm{CO}_{2}$ diminuiu com o aumento no teor de biodiesel na mistura (Figuras 1 e 2), independentemente do tipo de diesel. Entretanto, no diesel S10, houve 
maior redução $(0,03 \%)$ para o incremento de $10 \%$ de biodiesel. A regressão apresentou melhor ajuste com o uso do diesel S10, semelhantemente ao ocorrido com o $\mathrm{O}_{2}$. Houve redução de $4,5 \%$ com a inclusão de $100 \%$ de biodiesel na mistura, em comparação à inclusão de $5 \%$. Esse resultado é semelhante ao obtido por Gonzalez Maziero et al. (2006), que relataram redução de 4,8\% nos níveis de emissão de $\mathrm{CO}_{2}$ com o uso de biodiesel de girassol. Ozsezen et al. (2009) verificaram redução de $7 \%$ nas emissões, com o incremento da concentração de biodiesel na mistura combustível. Já McCarthy et al. (2011) observaram aumento de 6 a 18\% nas emissões de $\mathrm{CO}_{2}$ com a inclusão de diferentes tipos de biodiesel. Özener et al. (2014) também constataram aumento (17\%) nas emissões de $\mathrm{CO}_{2}$, ao utilizar a mistura $\mathrm{B} 50$, enquanto Valente et al. (2012) apontaram aumento de $8,5 \%$ com o uso da mistura com $50 \%$ de biodiesel de óleo de fritura.

Em termos absolutos, a redução na emissão de $\mathrm{CO}_{2}$ foi de $0,35 \%$, entre as misturas B5 e B100, o que foi uma variação menor do que a observada entre os diferentes modos de operação (Tabela 1). Em termos relativos, houve redução de 4,6\% com o uso do B100, quando comparado ao B5. Esse resultado difere do relatado por Canakci \& Van Gerpen (2003), que registraram aumento nos níveis de emissão de $\mathrm{CO}_{2}$, de 1,8 e 1,2\%, quando trabalharam com biodiesel de soja e gordura animal, respectivamente.

$\mathrm{O}$ modo de operação que mais emitiu $\mathrm{CO}_{2}$ foi o M5, seguido dos modos M1, M6, M2, M7, M3, M4 e M8. Todos estes modos diferiram entre si pelo teste de Tukey. De forma análoga ao observado com o $\mathrm{O}_{2}$, o aumento na carga aplicada e a consequente redução na admissão de ar pelo motor resultaram em maior emissão de $\mathrm{CO}_{2}$. Dependendo do modo de operação, as emissões de $\mathrm{CO}_{2}$ estiveram entre 8,3 e 1,5\% (Tabela 1), o que representa amplitude de variação de $6,8 \%$. Já a amplitude das emissões, de acordo com os diferentes combustíveis analisados (Figuras 1 e 2), foi de 0,35\%. Dessa maneira, a influência do modo de operação foi $\sim 19$ vezes maior do que a do tipo de combustível utilizado.

As emissões de $\mathrm{NO}$ e $\mathrm{NO}_{2}$ geralmente são representadas, na literatura, por emissão de $\mathrm{NO}_{\mathrm{x}}$. Cerca de $95 \%$ dessas emissões são relativas aos monóxidos e $5 \%$ aos dióxidos. No presente trabalho, enquanto as emissões de NO tiveram média de $1.250 \mathrm{ppm}$, as de $\mathrm{NO}_{2}$ foram de $80 \mathrm{ppm}$.
De modo geral, houve diminuição nos níveis de NO com o aumento na concentração de biodiesel na mistura, independentemente do tipo de diesel usado (Figuras 1 e 2). Com o diesel S10, houve redução de 4,5 ppm na emissão de NO com o aumento de $10 \%$ na concentração de biodiesel (Figura 1). Já para o diesel S500, essa redução foi de 6,9 ppm (Figura 2). Independentemente do tipo de diesel, houve redução de $\sim 100$ ppm nas emissões de NO com o uso do $\mathrm{B} 100$, em comparação ao B5, o que representou redução de 6\%. McCarthy et al. (2011) constataram reduções entre 14 e $17 \%$ nas emissões de NO com o uso de diferentes tipos de biodiesel. Por sua vez, Zhu et al. (2011) afirmam que a diminuição nos níveis de emissão de $\mathrm{NO}_{\mathrm{x}}$ pela inclusão de biodiesel são mais acentuadas com o uso de diesel com baixo teor de enxofre. Fang et al. (2008), ao trabalhar com um diesel mineral muito semelhante ao S10 brasileiro, observaram que o uso de biodiesel puro diminuiu em $13 \%$ as emissões de $\mathrm{NO}_{\mathrm{x}}$. Conforme estes autores, há grande redução na emissão de $\mathrm{NO}_{\mathrm{x}}$ quando a combustão é realizada em menor temperatura. Contudo, na literatura, verifica-se que as emissões de $\mathrm{NO}_{\mathrm{x}}$ aumentam com o incremento de biodiesel na mistura. Gonzalez Maziero et al. (2006) relataram aumento de 5,7\% nos níveis de emissão de $\mathrm{NO}_{\mathrm{x}}$, quando utilizaram biodiesel de girassol.

$\mathrm{O}$ modo de operação também apresentou maior influência sobre as emissões de $\mathrm{NO}_{\mathrm{x}}$ do que o tipo de combustível. No caso do NO, elas variaram de 1.416,4 ppm, no M5, a 278 ppm no M4 (Tabela 1), o que representa redução de $80,3 \%$. Esse resultado pode ser explicado pela maior temperatura de combustão no M5. Valente et al. (2012) ressaltam que as emissões de $\mathrm{NO}_{\mathrm{x}}$ aumentam à medida que as cargas aplicadas ao motor diminuem.

A redução decorrente da inclusão do biodiesel foi de 100 ppm, e a dos diferentes modo de operação foi de $1.138,4$ ppm (Tabela 1). Assim, o modo de operação do motor tem uma participação $91,2 \%$ maior na alteração das emissões de NO, quando comparado ao tipo de combustível.

Quanto às emissões de $\mathrm{NO}_{2}$, elas ficaram entre $79 \mathrm{e}$ $81 \mathrm{ppm}$, com o uso do diesel S10, ou seja, praticamente não houve alteração pela adição do biodiesel (Figura 1). Houve aumento de apenas 0,2 ppm nas emissões de $\mathrm{NO}_{2}$ com a inclusão de $10 \%$ de biodiesel na mistura. Quando o diesel utilizado foi o S500, o comportamento 
das emissões de $\mathrm{NO}_{2}$ foi mais errático (Figura 2), com incremento médio de $0,5 \mathrm{ppm}$ nas emissões.

Com relação ao modo de operação, as emissões de $\mathrm{NO}_{2}$ diminuíram de 102,6 (M5) para 24,1 ppm (M8), isto é, houve $76,6 \%$ de redução. É pertinente salientar que as emissões de $\mathrm{NO}_{2}$ aumentaram com o incremento dos teores de biodiesel na mistura, enquanto as de NO diminuíram. Como as emissões de NO são mais representativas que as de $\mathrm{NO}_{2}$, as emissões de $\mathrm{NO}_{\mathrm{x}}$ diminuem com o aumento da participação de biodiesel. Independentemente do diesel utilizado e do modo de operação, houve redução de 5,6\% nas emissões de $\mathrm{NO}_{\mathrm{x}}$ quando se utilizou B100, em comparação ao B5.

\section{Conclusões}

1. Quanto maior o teor de biodiesel na mistura com diesel, menores são as emissões de $\mathrm{CO}_{2}, \mathrm{NO}_{2}, \mathrm{CO}, \mathrm{NO}$ e de hidrocarbonetos (HC).

2. Apenas as emissões de HC são influenciadas pelo tipo de combustível.

3. As emissões provenientes do motor agrícola diminuem com a redução da carga aplicada ao motor e com o aumento do teor de biodiesel na mistura com diesel.

4. O combustível diesel S10, com $20 \%$ de biodiesel, reduz as emissões e mantém o desempenho do motor, em comparação ao diesel comercial (S500).

\section{Agradecimentos}

Ao Conselho Nacional de Desenvolvimento Científico e Tecnológico $(\mathrm{CNPq})$ e à Fundação de Amparo à Pesquisa do Estado do Rio Grande do Sul (Fapergs), pela concessão de bolsas; e à Olfar: Alimento e Energia, pela doação do biodiesel utilizado no experimento.

\section{Referências}

ASSOCIAÇÃO BRASILEIRA DE NORMAS TÉCNICAS. NBR ISO 8178-4: motores alternativos de combustão interna - medição das emissões de gases de exaustão. Parte 4: ciclos de ensaio em regime constante para diferentes aplicações do motor. Rio de Janeiro: ABNT, 2012.

BALLESTEROS, R.; GUILLÉN-FLORES, J.; MARTÍNEZ, J.D. Carbonyl emission and toxicity profile of diesel blends with an animal-fat biodiesel and a tire pyrolysis liquid fuel. Chemosphere, v.96, p.155-166, 2014. DOI: 10.1016/j.chemosphere.2013.10.017.
BERNARDES, M.A. dos S. (Ed.). Environmental impact of biofuels. Rijeka: InTech, 2011. 280p. DOI: 10.5772/960.

BOLDAJI, M.T.; EBRAHIMZADEH, R.; KHEIRALIPOUR, K.; BORGHEI, A.M. Effect of some BED blends on the equivalence ratio, exhaust oxygen fraction and water and oil temperature of a diesel engine. Biomass and Bioenergy, v.35, p.4099-4106, 2011. DOI: 10.1016/j.biombioe.2011.02.055.

BRIJESH, P.; SREEDHARA, S. Exhaust emissions and its control methods in compression ignition engines: a review. International Journal of Automotive Technology, v.14, p.195-206, 2013. DOI: 10.1007/s12239-013-0022-2.

BUYUKKAYA, E. Effects of biodiesel on a DI diesel engine performance, emission and combustion characteristics. Fuel, v.89, p.3099-3105, 2010. DOI: 10.1016/j.biombioe.2011.02.055.

CANAKCI, M. Combustion characteristics of a turbocharged DI compression ignition engine fueled with petroleum diesel fuels and biodiesel. Bioresource Technology, v.98, p.1167-1175, 2007. DOI: $10.1016 /$ j.biortech.2006.05.024.

CANAKCI, M.; VAN GERPEN, J.H. Comparison of engine performance and emissions for petroleum diesel fuel, yellow grease biodiesel, and soybean oil biodiesel. Transactions of the ASAE, v.46, p.937-944, 2003. DOI: 10.13031/2013.13948.

CHAUHAN, B.S.; KUMAR, N.; CHO, H.M.; LIM, H.C. A study on the performance and emission of a diesel engine fueled with Karanja biodiesel and its blends. Energy, v.56, p.1-7, 2013. DOI: 10.1016/j.energy.2013.03.083.

DWIVEDI, G.; JAIN, S.; SHARMA, M.P. Impact analysis of biodiesel on engine performance: a review. Renewable and Sustainable Energy Reviews, v.15, p.4633-4641, 2011. DOI: 10.1016/j.rser.2011.07.089.

FANG, T.; LIN, Y.-C.; FOONG, T.M.; LEE, C.-F. Reducing NOx emissions from a biodiesel-fueled engine by use of low-temperature combustion. Environmental Science and Technology, v.42, p.8865-8870, 2008. DOI: 10.1021/es8001635.

GOKALP, B.; BUYUKKAYA, E.; SOYHAN, H.S. Performance and emissions of a diesel tractor engine fueled with marine diesel and soybean methyl ester. Biomass and Bioenergy, v.35, p.3575-3583, 2011. DOI: 10.1016/j.biombioe.2011.05.015.

GONZALEZ MAZIERO, J.V.G.; CORRÊA, I.M.; TRIELLI, M.A.; BERNARDI, J.A.; D'AGOSTINI, M.F. Avaliação de emissões poluentes de um motor diesel utilizando biodiesel de girassol como combustível. Engenharia na Agricultura, v.14, p.287-292, 2006.

KOÇAK, M.S.; ILERI, E.; UTLU, Z. Experimental study of emission parameters of biodiesel fuels obtained from canola, hazelnut, and waste cooking oils. Energy and Fuels, v.21, p.3622-3626, 2007. DOI: 10.1021/ef0600558.

KOHLHEPP, G. Análise da situação da produção de etanol e biodiesel no Brasil. Estudos Avançados, v.24, p.223-253, 2010. DOI: 10.1590/S0103-40142010000100017.

MCCARTHY, P.; RASUL, M.; MOAZZEM, S. Analysis and comparison of performance and emissions of an internal combustion engine fuelled with petroleum diesel and different bio-diesels. Fuel, v.90, p.2147-2157, 2011. DOI: 10.1016/j.fuel.2011.02.010.

Pesq. agropec. bras., Brasília, v.50, n.12, p.1168-1176, dez. 2015 DOI: $10.1590 / \mathrm{S} 0100-204 X 2015001200006$ 
MENDIBURU, F. de. Agricolae: statistical procedures for agricultural research. Version 1.1-1. 2012. Available at: <http:// tarwi.lamolina.edu.pe/ fmendiburu/>. Accessed on: 8 Oct. 2015.

ÖZENER, O.; YÜKSEK, L.; ERGENÇ, A.T.; ÖZKAN, M. Effects of soybean biodiesel on a DI diesel engine performance, emission and combustion characteristics. Fuel, v.115, p.875-883, 2014. DOI: $10.1016 /$ j.fuel.2012.10.081.

OZSEZEN, A.N.; CANAKCI, M.; TURKCAN, A.; SAYIN, C. Performance and combustion characteristics of a DI diesel engine fueled with waste palm oil and canola oil methyl esters. Fuel, v.88, p.629-636, 2009. DOI: 10.1016/j.fuel.2008.09.023.

R DEVELOPMENT CORE TEAM. R: a language and environment for statistical computing. Vienna, Austria: R Foundation for Statistical Computing, 2012. ISBN 3-900051-07-0.

SAHOO, P.K.; DAS, L.M.; BABU, M.K.G.; ARORA, P.; SINGH, V.P.; KUMAR, N.R.; VARYANI, T.S. Comparative evaluation of performance and emission characteristics of jatropha, karanja and polanga based biodiesel as fuel in a tractor engine. Fuel, v.88, p.1698-1707, 2009. DOI: 10.1016/j.fuel.2009.02.015.

SANJID, A.; MASJUKI, H.H.; KALAM, M.A.; RAHMAN, S.M.; ABEDIN, M.J.; PALASH, S.M. Production of palm and jatropha based biodiesel and investigation of palm-jatropha combined blend properties, performance, exhaust emission and noise in an unmodified diesel engine. Journal of Cleaner Production, v.65, p.295-303, 2014. DOI: 10.1016/j. jclepro.2013.09.026.

VALENTE, O.S.; PASA, V.M.D.; BELCHIOR, C.R.P.; SODRÉ, J.R. Exhaust emissions from a diesel power generator fuelled by waste cooking oil biodiesel. Science of the Total Environment, v.431, p.57-61, 2012. DOI: 10.1016/j.scitotenv.2012.05.025.

ZHU, R.; CHEUNG, C.S.; HUANG, Z.; WANG, X. Regulated and unregulated emissions from a diesel engine fueled with diesel fuel blended with diethyl adipate. Atmospheric Environment, v.45, p.2174-2181, 2011. DOI: 10.1016/j.atmosenv.2011.01.062.

Recebido em 25 de fevereiro de 2015 e aprovado em 9 de outubro de 2015 